\title{
The Spectrum of Kidney Disease in Type Two Diabetic Patients: A Single-Center Study
}

\author{
Amir Muse Mohamud1,2, Ning Xu1,2, Guangyi Liu',2, Bekzod Odilov,4, Bei Jiang1,2, Zhao Hu1,2* \\ ${ }^{1}$ Department of Nephrology, School of Medicine, Cheeloo College of Medicine, Shandong University, Jinan, China \\ ${ }^{2}$ Department of Nephrology, Qilu Hospital, Cheeloo College of Medicine, Shandong University, Jinan, China \\ ${ }^{3}$ Department of Endocrinology, School of Medicine, Cheeloo College of Medicine, Shandong University, Jinan, China \\ ${ }^{4}$ Department of Endocrinology, Qilu Hospital, Cheeloo College of Medicine, Shandong University, Jinan, China \\ Email: *sdhuzhao@163.com,dramirmuse@gmail.com
}

How to cite this paper: Mohamud, A.M., Xu, N., Liu, G.Y., Odilov, B., Jiang, B. and $\mathrm{Hu}, \mathrm{Z}$. (2022) The Spectrum of Kidney Disease in Type Two Diabetic Patients: A Single-Center Study. Open Journal of Nephrology, 12, 1-14.

https://doi.org/10.4236/ojneph.2022.121001

Received: December 15, 2021

Accepted: January 10, 2022

Published: January 13, 2022

Copyright $\odot 2022$ by author(s) and Scientific Research Publishing Inc. This work is licensed under the Creative Commons Attribution International License (CC BY 4.0).

http://creativecommons.org/licenses/by/4.0/

\begin{abstract}
Background: Diabetic nephropathy (DN) is the dominant reason for end-stage kidney disease linked with a rise in cardiovascular mortality rate. However, besides DN, type 2 diabetic patients may also suffer from various non-diabetic renal diseases (NDRD). Aim: The objective of the current research was to assess the occurrence and type of NDRD diagnosed by kidney biopsy in type 2 diabetic subjects, evaluate the association of various clinical and laboratory characteristics with histopathology findings, and identify essential predictors of NDRD. Methods: Retrospective analysis has been performed through medical record revision of 101 patients with type 2 diabetes undergoing percutaneous renal biopsy at Qilu Hospital of Shandong University (Jinan, China) between January 2015 and December 2020. Results: Renal biopsy results showed that NDRD was found in 59 patients (58.42\%), while DN existed in 32 patients $(31.68 \%)$ and 10 patients $(9.90 \%)$ showed DN complicated with NDRD. Membranous nephropathy was prevailing NDRD (42\%), followed by focal segmental glomerulosclerosis (11.6\%) and IgA nephropathy (10.1\%). In univariate analysis, patients with NDRD had older age $(\mathrm{p}<0.018)$, a short duration of diabetes $(\mathrm{p}<0.000)$, lower proteinuria $(\mathrm{p}<0.030)$, and had higher hemoglobin levels $(\mathrm{p}<0.006)$ compared to non-NDRD patients. In multivariate logistic regression analysis, the short course of diabetes (OR 0.986; $95 \% \mathrm{CI}=0.978-0.993 ; \mathrm{p}=0.000)$ and older age $(\mathrm{OR} 1.080 ; 95 \% \mathrm{CI}=1.028-$ 1.134; $\mathrm{p}=0.002)$ were significant risk factors for NDRD occurrence. In ROC analysis for NDRD, the duration of diabetes $\leq 78$ months (cut-off value $(0.725$, 0.313)) illustrated the highest AUC. Conclusions: Clinical parameters such as short duration of diabetes, older age, higher hemoglobin level, and lower proteinuria might be associated with NDRD in type 2 diabetic patients. An early diagnosis of NDRD poses a favorable renal prognosis because it requires
\end{abstract}


a different approach than $\mathrm{DN}$, further larger multicenter randomized prospective investigations focused on identifying possible risk markers of NDRD are still in priority.

\section{Keywords}

Type-Two Diabetes Mellitus, Non-Diabetic Renal Disease, Diabetic Nephropathy, Kidney Biopsy

\section{Introduction}

Diabetes mellitus (DM), which is considered a public health threat, affects various organs and results in different vascular and non-vascular complications. The prevalence of DM is showing a significant upward trend worldwide in recent years [1]. In 2015, more than 410 million people were estimated as being diabetic; by 2040 , this number is predicted to outweigh 640 million, with rising disproportionately in low- to middle-income countries [2] with an increasingly aging population and global obesity pandemic is the urging trigger behind the rising occurrence of diabetes [3] [4]. The most current national proportion of diabetic adults in the United States is $12.2 \%$ [5]. The total estimated occurrence of diabetes in China in 2017 was approximately 13\% using the ADA criteria for diagnosis and around 11\% according to World Health Organization criteria [6]. As reported by the diabetes atlas of the IDF, the world's largest diabetic population resides in China. Estimations indicate that China has 113.9 million adults living with diabetes, making almost one-fourth of all diabetes patients globally [7].

Type 2 diabetes mellitus is one of the most relevant risk factors for CKD development; approximately $30 \%-50 \%$ of ESRD patients come from a diabetic origin [8].

Diabetic nephropathy is causing an increase in the number of patients with ESKD as a result of a steady rise in the prevalence of diabetes mellitus (DM); especially type $2 \mathrm{DM}$, which is ten times more prevalent than type 1 , as well as an increase in the life expectancy of diabetic patients who develop such late complications [9]. The underlying pathogenetic components of the onset and development of renal injury in diabetic nephropathy are multifactorial. Renal hemodynamic abnormalities, such as increased glomerular filtration rate (GFR), hydrostatic capillary pressure, and persistent hyperglycemia, cause biochemical shifts in the polyol pathway and contribute to the production of advanced nonenzymatic glycosylation products, are among them [10]. However, new evidence is emerging that the immune response represents a crucial impact on diabetic nephropathy progression [8]. Although each cell type in the glomerular tuft is damaged by diabetic nephropathy, damage to podocytes is considered as a key to the advancement of glomerulosclerosis [11] [12]. DN is not the isolated type of kid- 
ney damage in diabetic patients, as other damage types apart from diabetic nephropathy are accumulatively considered a non-diabetic renal disease (NDRD) [13]. In documented studies [1] [13]-[27], the prevalence of biopsy-proven NDKD in T2DM varies greatly, varying between $8 \%$ [14] and 93.5\% [15]. This percentage is based on the population examined and selected for kidney biopsy, together with the accessibility of renal biopsy. NDRD, compared to DN, includes a wide range of kidney injuries. Among DN and NDRD, there are important clinical, pathological features and prognostic heterogeneity. The clinical and pathological diagnosis of these diseases does have a major impact on the treatment options available and the patients' renal prognosis. Even so, there is no uniformity in the way these patients are diagnosed. Diagnosis of DN commonly depends on clinical signs and is supported by the presence of hypertension, persistent proteinuria, and continuous decrease in renal functionality [28], whilst the kidney biopsy is not absolutely compulsory. Patients affected by NDRD, in contrast remain misdiagnosed.

Treatment approaches of DN and NDRD differ noticeably. It has been widely assumed that DN reversal is hard to achieve. On the other hand, some forms of NDRD are easily curable and even remittable. To the common knowledge, kidney biopsy is the most crucial diagnostic tool in differentiating DN and NDRD. Although the procedure of renal biopsy is accompanied by complications, such as relatively large trauma and bleeding, it is usually recommended to do a renal biopsy in patients matching NDRD indications. Nevertheless, there is still controversy whether it is important to perform kidney biopsy if patients with diabetes represent more kidney disease-related symptoms. Therefore, further research is required to identify NDRD predictors.

The current study was designed to determine the occurrence and type of nondiabetic renal disease diagnosed by kidney biopsy in type 2 diabetic patients, examine possible association of clinical and laboratory parameters with histopathology findings, as well as identifying essential predictors of NDRD.

\section{Methods}

\subsection{Study Population}

Initially, 200 type 2 diabetic patients who had undergone percutaneous kidney biopsy at Qilu Hospital of Shandong University (Jinan, China) from January 2015 to December 2020 were included for this research. Exclusion criteria for current study were following: type 1 diabetic patients; type 2 diabetic patients with missing information or unclear medical history (such as, missing fundus ophthalmoscopy); patients with current acute illnesses (urinary tract infection, respiratory tract infection, digestive tract infection, etc.) and active malignancy. After removing subjects who have met exclusion criteria, 101 patients were enrolled in our research. At the time of the biopsy, all subjects were diagnosed with type 2 diabetes according to WHO [29] and ADA [30] criteria. Indications for biopsy were consistently based on clinically suspected NDRD in accordance 
with one or more of the criteria listed below: Persistent glomerular hematuria, rapid onset of heavy proteinuria, absence of diabetic retinopathy, and unexplained rapidly progressive renal failure.

At the time of the biopsy, the patient's age, gender, duration of diabetes prior to the renal biopsy, evidence of hypertension, and presence of diabetic retinopathy were recorded. Additionally, laboratory parameters such as serum creatinine level and estimated glomerular filtration rate (eGFR) get calculated by using the chronic kidney disease epidemiology collaboration (CKD-EPI) formula [31], Fasting blood glucose, glycated hemoglobin A1C (HbA1c), serum albumin, hemoglobin level, anti-nuclear antibody (ANA), erythrocyte sedimentation rate (ESR), presence of proteinuria, presence of glomerular hematuria also were obtained. Diabetes duration was defined as the interval between diabetes onset and kidney biopsy, and patients with systolic blood pressure $\geq 140 \mathrm{mmHg}$ or diastolic blood pressure $\geq 90 \mathrm{mmHg}$ or using antihypertensive was diagnosed with hypertension. An ophthalmologist diagnosed diabetic retinopathy by performing direct ophthalmoscopy. Hematuria was characterized as the presence of $>11$ red blood cells/ $\mu$ l using phase-contrast urine microscopy. Proteinuria was quantified using a spot urine protein-to-creatinine ratio. The Ethical Committee of Qilu Hospital of Shandong University has approved current research and all subjects have provided written consent prior to renal biopsy. All cases have been diagnosed by using light microscopy, immunofluorescence, and electron microscopy. Two skilled and independent pathologists examined all biopsy samples. Pathological diagnostic findings of diabetic nephropathy (DN) were mesangial expansion, basement membrane thickenings, and diffuse intercapillary glomerulosclerosis with or without Kimmelstiel-Wilson nodules, as well as exudative lesions, like fibrin caps, hyaline thrombi, or capsular drop [32] while NDRDs was diagnosed following orthodox criteria [33]. According to their pathology reports, patients were grouped into three: a group of isolated DN patients, a group of DN complicated by NDRD (Mixed) patients, and a group of isolated NDRD patients. Moreover, an additional classification category was formed based on the presence of NDRD (NDRD vs. non-NDRD patients).

\subsection{Statistical Analysis}

IBM SPSS Statistics version 26 was used for statistical analysis. Normally distributed data were expressed as mean \pm standard deviation; skewed data as median with interquartile range, categorical data were reported as frequency (\%). For evaluating the differences between groups, a Student $t$-test or ANOVA was used for normally distributed data, while Mann-Whitney U test or KruskalWallis test was performed for skewed data, and the chi-square $\left(\chi^{2}\right)$ test was used for categorical data. The stepwise forward method has been used in multivariate (binary) logistic regression to determine independent predictors of DN and NDRD, using all factors having a $\mathrm{p}$-value of $<0.05$ in univariate analysis. By plotting sensitivity vs. 1-specificity for significant variables in NDRD, receiv- 
er operating characteristic (ROC) curves were formed, and the areas under the ROC curves (AUC) were calculated to determine the sensitivity and specificity of predictors. The $\mathrm{p}$-value of $<0.05$ was considered statistically significant during analysis.

\section{Results}

This study enrolled 101 type-two diabetic patients. The median age at biopsy was $51.2 \pm 10.9$ years, $41 \%$ of the patients (41) were female, and the average duration of diabetes was four years (varying between 1 month and 300 months). Table 1 summarizes baseline clinical and laboratory parameters of patients.

As reported by kidney biopsy, NDRD was found in 59 patients (58.42\%), while isolated DN was diagnosed in 32 patients (31.68\%), and DN complicated with NDRD was present in the remaining ten patients (9.90\%). As indicated in Table 2, focal segmental glomerulosclerosis (FSGS) was found in 8 patients (11.6\%) and IgA nephropathy in 7 patients (10.1\%), while membranous nephropathy, which was diagnosed in 29 patients $(42 \%)$, remained the most prevalent NDRD.

Moreover, univariate analysis of primary classification groups (DN vs. NDRD vs. mixed lesions (in Table 1) revealed a significant variation in terms of age ( $\mathrm{p}$ $<0.030)$, diabetes duration $(\mathrm{p}<0.001)$, and hemoglobin $(\mathrm{p}<0.006)$. Alongside

Table 1. Clinical and biochemical characteristics of the study patients in total and in classification groups (Isolated DN vs. Mixed lesions vs. Isolated NDRD).

\begin{tabular}{|c|c|c|c|c|}
\hline & $\operatorname{ALL}(\mathrm{n}=101)$ & $\mathrm{DN}(\mathrm{n}=32)$ & $\operatorname{MIX}(\mathrm{n}=10)$ & $\operatorname{NDRD}(\mathrm{n}=59)$ \\
\hline Age (years)* & $51.2 \pm 10.9$ & $47.4 \pm 10.7$ & $49.2 \pm 13.9$ & $53.56 \pm 9.95$ \\
\hline Gender (Male) & $60(59 \%)$ & $23(71.9 \%)$ & $9(90 \%)$ & $28(47 \%)$ \\
\hline Diabetes duration (Months) ${ }^{\star}$ & $48(114-12)$ & $96(177-48)$ & $30(135-6)$ & $36(96-11.5)$ \\
\hline $\mathrm{FBG}(\mathrm{mmol} / \mathrm{l})$ & $6.36(8.19-5.20)$ & $5.97(8.44-5)$ & $6.32(8.26-4.89)$ & $6.44(7.87-5.38)$ \\
\hline Hemoglobin Alc (\%) & $6.8(8.6-6.1)$ & $7(9.45-6.4)$ & $6.45(9.91-5.97)$ & $6.8(7.90-6.10)$ \\
\hline Proteinuria (g/g) & $4.18(8.05-1.62)$ & $6.22(10.20-3.13)$ & $4.20(9.84-0.85)$ & $3.30(7.35-1.37)$ \\
\hline Serum albumins $(\mathrm{g} / \mathrm{l})$ & $31.95 \pm 7.91$ & $33.40 \pm 6.47$ & $29.42 \pm 6.54$ & $31.59 \pm 8.75$ \\
\hline Serum creatinine $(\mathrm{mmol} / \mathrm{l})$ & $99(165-67)$ & $116(182-72)$ & $133(224.75-79)$ & $84(154-58)$ \\
\hline Estimated GFR (ml/minute) & $66.96(106.26-40.62)$ & $59.93(97.16-35.90)$ & $50.42(68.95-33.98)$ & $84.06(112.75-41.04)$ \\
\hline Hemoglobin $(\mathrm{g} / \mathrm{l})$ * & $122(134.5-107)$ & $116(126.5-101.25)$ & $113(132.5-95.5)$ & $127(143-114)$ \\
\hline $\operatorname{ESR}(\mathrm{mm} / \mathrm{hr})$ & $43(71-27)$ & $47.5(67.8-28.25)$ & $47.5(83.5-39.5)$ & $39(71-18)$ \\
\hline ANA $\geq 1: 80$ (dilution) & $18(18 \%)$ & $3(9 \%)$ & $2(20 \%)$ & $13(22 \%)$ \\
\hline Hypertension & $69(68 \%)$ & $24(75 \%)$ & $4(40 \%)$ & $41(69.5 \%)$ \\
\hline Hematuria & $43(43 \%)$ & $17(53 \%)$ & $4(20 \%)$ & $24(40.7 \%)$ \\
\hline Diabetic retinopathy & $55(54.5 \%)$ & $18(56.3 \%)$ & $8(80 \%)$ & $29(49.2 \%)$ \\
\hline
\end{tabular}

${ }^{*} \mathrm{p}<0.05$ (ANOVA/Kruskal-Wallis-test/ $\chi{ }^{2}$-test/Fisher's-test). FBG, fasting blood glucose; GFR, glomerular filtration rate; DN, diabetic nephropathy; NDRD, Non-diabetic renal disease; MIX, NDRD superimposed on DN. 
Table 2. Pathological findings of NDRD with or without diabetic nephropathy.

\begin{tabular}{cccc}
\hline Type of NDRD & All (n=69) & MIX (n=10) & NDRD (n = 59) \\
\hline Membranous nephropathy & $29(42 \%)$ & $4(40 \%)$ & $25(42.4 \%)$ \\
Focal segmental glomerulosclerosis & $8(11.6 \%)$ & $1(10 \%)$ & $7(11.9 \%)$ \\
IgA nephropathy & $7(10.1 \%)$ & 0 & $7(11.9 \%)$ \\
Minimal change disease & $6(8.7 \%)$ & $1(10 \%)$ & $5(8.5 \%)$ \\
Tubulointerstitial nephritis & $5(7.3 \%)$ & $1(10 \%)$ & $4(6.8 \%)$ \\
Renal amyloidosis & $2(2.9 \%)$ & 0 & $2(3.4 \%)$ \\
Lupus nephritis & $2(2.9 \%)$ & $1(10 \%)$ & $1(1.7 \%)$ \\
Hypertensive renal damage & $2(2.9 \%)$ & $1(10 \%)$ & $1(1.7 \%)$ \\
$\begin{array}{c}\text { Hepatitis B virus associated } \\
\text { glomerulonephritis }\end{array}$ & $2(2.9 \%)$ & $1(10 \%)$ & $1(1.7 \%)$ \\
$\begin{array}{c}\text { ANCA associated glomerulonephritis } \\
\begin{array}{c}\text { Immune mediated glomerulonephritis } \\
\text { Light chain deposition disease }\end{array}\end{array}$ & $1(1.45 \%)$ & 0 & $1(1.7 \%)$ \\
$\begin{array}{c}\text { Crescentic glomerulonephritis with } \\
\text { Tubulointerstitial nephritis }\end{array}$ & $1(1.45 \%)$ & 0 & $1(1.7 \%)$ \\
$\begin{array}{c}1(1.45 \%) \\
\text { chronic sclerosing glomerulosclerosis } \\
\text { with interstitial arteriosclerosis }\end{array}$ & $1(1.45 \%)$ & 0 & $1(1.7 \%)$ \\
Acute interstitial nephritis & $1(1.45 \%)$ & 0 & $1(1.7 \%)$ \\
\hline
\end{tabular}

NDRD, non-diabetic renal disease; MIX, NDRD superimposed on diabetic nephropathy.

the used univariate analysis also revealed that subjects with NDRD had older age $(\mathrm{p}<0.018)$, shorter duration of diabetes $(\mathrm{p}<0.000)$, lower proteinuria $(\mathrm{p}<0.030)$, and had higher hemoglobin levels $(\mathrm{p}<0.006)$ compared to non-NDRD patients, as shown in (Table 3).

To identify risk factors for NDRD, multivariate (binary) logistic regression analysis was performed, and variables considered statistically significant in univariate analysis were included. The findings are outlined in Table 4.

We also found that the short diabetes duration (OR 0.986; 95\% CI $=0.978$ $0.993 ; \mathrm{p}=0.000)$ and older age (OR 1.080;95\% CI $=1.028-1.134 ; \mathrm{p}=0.002)$ were significant NDRD risk factors. The sensitivity and specificity of risk factors associated with NDRD were evaluated using ROC analysis, and the results are shown in Table 5.

Table 5 Sensitivity, Specificity, Positive and Negative Predictive Values of Significant Variables in The Prediction of Diabetic Kidney Disease and of NonDiabetic Renal Disease.

The duration of diabetes less than or equal to 78 months (cut-off value $(0.725$, 0.313)) showed the highest AUC for NDRD. Figure 1 illustrates the AUC curves for the predictors of NDRD. 
Table 3. Clinical and biochemical characteristics of the study patients in the classification group (NDRD vs. Non-NDRD).

\begin{tabular}{cccc}
\hline & NDRD $(\mathrm{n}=69)$ & non-NDRD (n32) & p-Value \\
\hline Age (years)* & $52.9 \pm 10.61$ & $47.44 \pm 10.67$ & 0.018 \\
Gender (Male) & $37(53.6 \%)$ & $23(71.9 \%)$ & 0.082 \\
Diabetes duration (Months)* & $36(96-11)$ & $96(177-48)$ & 0.000 \\
FBG (mmol/l) & $6.44(7.98-5.37)$ & $5.97(8.44-5)$ & 0.504 \\
Hemoglobin A1c (\%) & $6.8(7.95-6.10)$ & $7(9.45-6.4)$ & 0.361 \\
Proteinuria (g/g)* & $3.34(7.69-1.36$ & $6.22(10.20-3.13)$ & 0.030 \\
Serum albumins (g/l) & $31.951 \pm 8.46$ & $33.40 \pm 6.47$ & 0.209 \\
Serum creatinine (mmol/l) & $87(157-63.5)$ & $116(182-72)$ & 0.198 \\
Estimated GFR (ml/minute) & $79.2(112.6-40.9)$ & $59.93(97.16-35.90)$ & 0.356 \\
Hemoglobin (g/l)* & $127(142.5-113)$ & $116(126.5-101.25)$ & 0.006 \\
ESR (mm/hr) & $40(72-19.5)$ & $47.5(67.8-28.25)$ & 0.614 \\
ANA $\geq 1: 80$ (dilution) & $15(21.7)$ & $3(9.4 \%)$ & 0.131 \\
Hypertension & $45(65.2 \%)$ & $24(75 \%)$ & 0.326 \\
Hematuria & $26(37.7 \%)$ & $17(53 \%)$ & 0.144 \\
Diabetic retinopathy & $37(53.6 \%)$ & $18(56.3 \%)$ & 0.805 \\
\hline
\end{tabular}

${ }^{\star} \mathrm{p}<0.05$ (ANOVA/Mann-Whitney-test/ $\chi^{2}$-test/Fisher's-test). FBG, fasting blood glucose; GFR, glomerular filtration rate; DN, diabetic nephropathy; NDRD, non-diabetic renal disease; MIX, NDRD superimposed on DN.

Table 4. Multivariate logistic regression analysis of diabetic nephropathy and of non-diabetic renal disease.

\begin{tabular}{ccccccc}
\hline Indicator & $\beta$-Estimate & Standard error & Wald $\left(\chi^{2}\right)$ p-Value & OR & $95 \%$ CI \\
\hline \multicolumn{7}{c}{ for NDRD } \\
\hline $\begin{array}{c}\text { Diabetes duration } \\
\text { (Months) }\end{array}$ & -0.014 & 0.004 & 13.399 & 0.000 & 0.986 & $0.978-0.993$ \\
Age (years) & 0.077 & 0.025 & 9.366 & 0.002 & 1.080 & $1.028-1.134$ \\
\hline
\end{tabular}

NDRD, non-diabetic renal disease; OR, odds ratio; CI, confidence interval.

Table 5. Sensitivity, specificity, positive and negative predictive values of significant variables in the prediction of diabetic kidney disease and of non-diabetic renal disease.

\begin{tabular}{ccccccc}
\hline Prediction of NDRD & AUC & $\begin{array}{c}\text { Sensitivity } \\
(\%)\end{array}$ & $\begin{array}{c}\text { Specificity } \\
(\%)\end{array}$ & PPV (\%) & NPV (\%) & p-Value \\
\hline $\begin{array}{c}\text { Duration of diabetes } \\
(\leq 78 \text { months })\end{array}$ & 0.737 & 72.46 & 68.75 & 83.34 & 53.65 & 0.0001 \\
\begin{tabular}{c} 
Age ( $\geq 50.5$ years $)$ \\
\hline
\end{tabular} & 0.649 & 62.32 & 62.50 & 78.18 & 43.47 & 0.016 \\
\hline
\end{tabular}

NDRD, non-diabetic renal disease; AUC, area under curve; PPV, positive predictive value; NPV, negative predictive Value. 

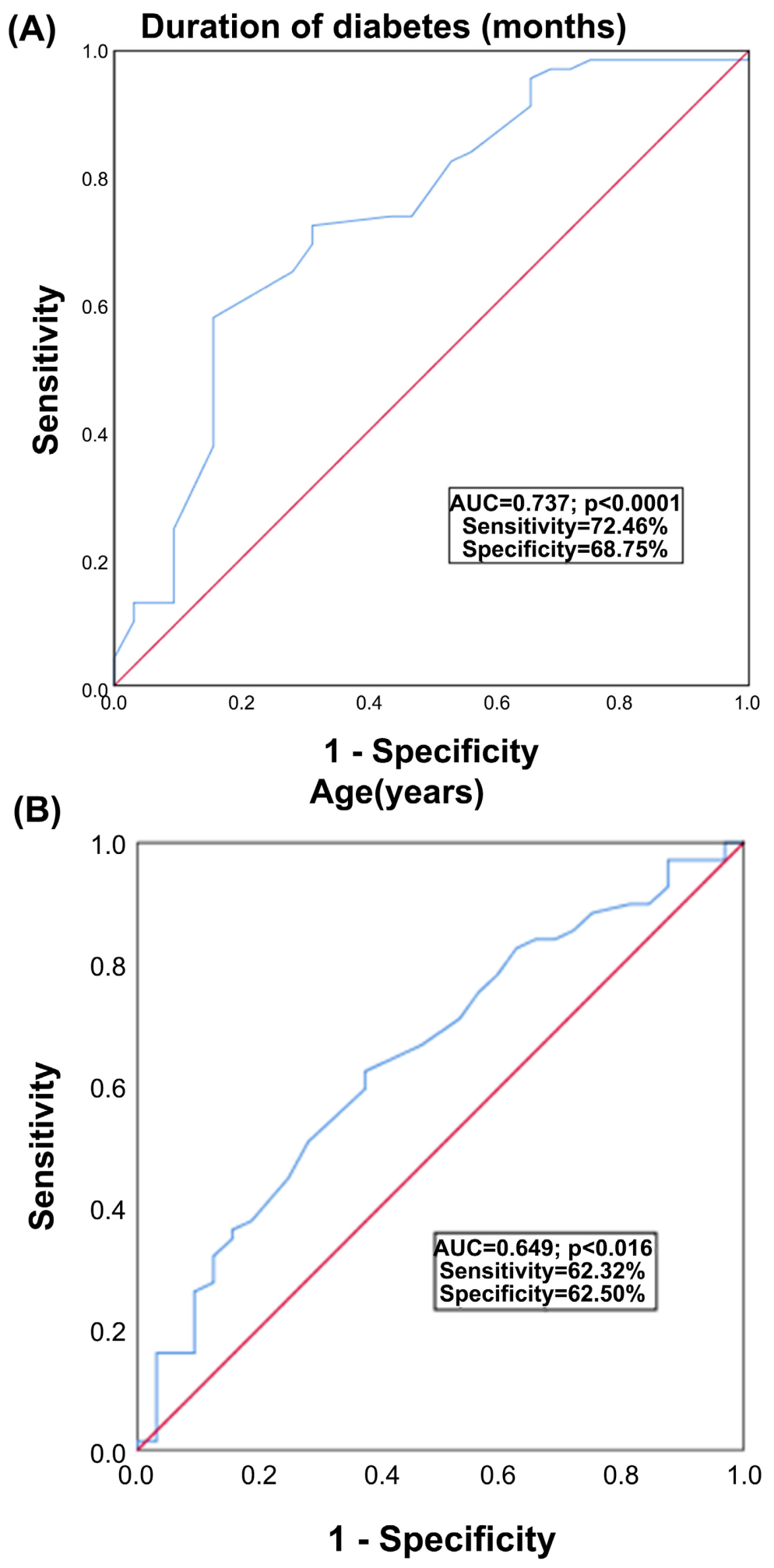

Figure 1. Receiver operating characteristic curves in the analysis of predictors of nondiabetic renal disease. (A) For shorter duration of diabetes, (B) For older age.

\section{Discussion}

Diabetic nephropathy (DN) is the dominant reason for end-stage kidney disease 
and correlates with a higher cardiovascular mortality rate [8]. However, Diabetic nephropathy is not the sole kidney pathology seen in patients with type 2 diabetes; a wide range of non-diabetic renal disease (NDRD), comprising both glomerular and tubulointerstitial invasions, may still present [34]. Previous studies [1] [13]-[27] [34]-[38] demonstrated that the prevalence of biopsy-proven NDKD in T2DM varies greatly, ranging from $8 \%$ [14] to $93.5 \%$ [15]. In our study, the prevalence was $68.32 \%$, most similar to Wilfred et al.'s findings [23]. The disparity in the prevalence of NDRD could be explained through demographic variations of participants included as well as dissimilar indications for biopsy. In current study, patients undergoing renal biopsy were defined by using prevalent indications which can be seen in other similar studies, namely, proteinuria [1] [13] [15] [16] [20], rapidly progressive renal failure [1] [15] [16] [17] [19] [24], glomerular hematuria [15] [18] [20] [24], absence of diabetic retinopathy [13] [15] [18] [19] [35] and shorter duration of diabetes [15] [16] [36] [39].

In our study, glomerulonephritis was the most prevalent type of NDRD, being similar to findings of majority studies [13] [15] [16], while interstitial nephritis was defined as prevailing NDRD according to some researches [16] [20]. We found that membranous nephropathy 29 (42\%) was the most prevalent NDRD, followed by focal segmental-glomerulosclerosis 8 (11.6\%), IgA Nephropathy 7 (10.1\%), minimal change disease 6 (8.7\%), tubulointerstitial Nephritis 5 (7.3\%), renal amyloidosis $2(2.9 \%)$, lupus nephritis 2 (2.9\%), hypertensive renal damage $2(2.9 \%)$ cases.

It is also noteworthy that, besides regional and ethnic variations, different approaches to renal pathological diagnosis may impact the pathological distribution of NDRD in diabetic patients. For instance, focal segmental glomerulosclerosis, IgA nephropathy, and membranoproliferative glomerulonephritis were the most prevalent type of NDRD in the USA, Korea, as well as in Iraq, while acute interstitial nephritis and post-infectious glomerulonephritis were identified more often in India [27].

Since NDRD requires additional treatment and poses an opportunity for renal function preservation, its detection remains principal [37]. Although renal biopsy is approximately the only tool that can definitively distinguish DN from NDRD, identification of NDRD related prognostic factors prior to biopsy is critical. Clinical parameters, such as shorter duration of diabetes, older age, lower proteinuria, and higher hemoglobin level were identified as significant predictors of NDRD in univariate analysis (NDRD vs. non-NDRD). However, only the duration of diabetes less than or equal to 78 months and age greater than or equal to 50.5 years were statistically significant independent risk factors of NDRD when multivariate analysis was performed.

Previous studies have identified the short diabetes duration as being a significant predictor of NDRD through univariate or multivariate analysis [17] [21] [22] [23] [38] [40]. Thus, our findings further support the concept that short diabetes duration may hint at the presence of NDRD and necessitate kidney biop- 
sy. In our study, the sensitivity and specificity of the short duration of diabetes in predicting NDRD was found to be $72.46 \%$ and $68.75 \%$, respectively; these results were comparable to the ones reported by Tone et al. discovered with the sensitivity and specificity of $75 \%$ and $70 \%$, respectively [21].

Previously, some studies identified older age being a risk factor of NDRD presence [26] [40]. In our study, the sensitivity and specificity of older age in predicting NDRD was found to be $62.32 \%$ and $62.50 \%$, respectively. This predictor's sensitivity and specificity have never been reported in published studies. Certainly, the association between the short duration of diabetes and NDRD was likely. However, the possibility of older age in diabetic patients as an NDRD risk factor has also been suggested by our findings. Thus, it may be reasonable to perform a renal biopsy in the case of suspicion, even in elderly diabetic patients.

In this study, we have not identified any other clinical or laboratory variable that was a statistically significant predictor of NDRD in multivariate analysis, although higher hemoglobin levels were significantly associated with NDRD in univariate analysis. Similarly, the significant findings illustrating the higher hemoglobin levels as NDRD predictor has not been found in other studies, except findings of Chang et al. [17] and M. Liu et al. [41], which also discovered statistical significance in multivariate analysis. It has been assumed that in patients with NDRD, hemoglobin level may correlate with lower serum creatinine levels. Moreover, anemia may occur earlier in patients suffering from diabetic nephropathy compared to patients with non-diabetic glomerular disease, and similar renal function may somehow result in this independent predictive power of the hemoglobin level on NDRD [42]. However, some studies have suggested that diabetic patients are generally affected by visceral nerve innervation disorders that potentially may cause defective erythropoietin (EPO) secretion. This evidence may support the significantly higher possibility of associated anemia in diabetic patients [43] [44].

Lower proteinuria, which had statistical significance in our univariate analysis, was also identified as significant predictors of NDRD in other research $(\mathrm{Bi}$, H. et al.) [20].

Identifying and monitoring $\mathrm{DN}$ is based on evaluations of renal function, generally with an estimated GFR (eGFR) of $<60 \mathrm{~mL} / \mathrm{min} / 1.73 \mathrm{~m}^{2}$ and renal damage, generally with albuminuria $>30 \mathrm{mg} / \mathrm{g}$ creatinine [45]. Several previous studies found that a long duration of diabetes may correlate with DN [16] [22]. In combination with this study results, the duration of diabetes mellitus is a significant predictor for NDRD and DN identification.

The main limitations of the current study include the following: it was a retrospective and monocentric study. Thus, biases in patient selection might lead to ascertainment error, recall, or lead-time biases. Even though only type 2 diabetic patients with a high suspicion of NDRD were enrolled, biases in the patient selection are another limitation of our study. As demonstrated, there was significant heterogeneity in the criteria used to conduct a renal biopsy, highlighting the importance of identifying clinical predictors of NDRD. The recog- 
nized NDRD indications were chosen as the criteria for renal biopsy. Furthermore, the anti-phospholipase A2 receptor antibody and body mass index were excluded from the analysis considering the high percentage of missed values. However, these limitations might make it difficult to draw definitive conclusions for the entire diabetic population.

\section{Conclusion}

To conclude, clinical parameters such as short duration of diabetes, older age, higher hemoglobin level, and lower proteinuria might be associated with NDRD in type 2 diabetic patients. Early diagnosis of NDRD poses a favorable renal prognosis because it requires a different approach than $\mathrm{DN}$, further larger multicenter randomized prospective investigations focused on identifying possible risk markers of NDRD are still in priority.

\section{Authors' Contributions}

Conceptualization, methodology, formal analysis, writing-original draft preparation, A.M.M.; data curation, formal analysis, N.X.; resources, Conceptualization, G.L.; writing-review and editing, software, formal analysis, B.O.; resources, visualization, B.J.; Conceptualization, supervision, project administration, funding acquisition, Z.H.

\section{Conflicts of Interest}

The authors declare no conflicts of interest.

\section{References}

[1] Zhou, J., et al. (2008) A Differential Diagnostic Model of Diabetic Nephropathy and Non-Diabetic Renal Diseases. Nephrology Dialysis Transplantation, 23, 1940-1945. https://doi.org/10.1093/ndt/gfm897

[2] Atlas, D. (2015) IDF Diabetes Atlas. 7th Edition, International Diabetes Federation, Brussels.

[3] Zhou, B., et al. (2016) Worldwide Trends in Diabetes since 1980: A Pooled Analysis of 751 Population-Based Studies with 4.4 Million Participants. The Lancet, 387, 1513-1530. https://doi.org/10.1016/S0140-6736(16)00618-8

[4] World Health Organization (2014) Global Status Report on Noncommunicable Diseases 2014.

[5] Freeman, N.S., Canetta, P.A. and Bomback, A.S. (2020) Glomerular Diseases in Patients with Diabetes Mellitus: An Underappreciated Epidemic. Kidney360, 1, 220 222. https://doi.org/10.34067/KID.0000792019

[6] Li, Y., et al. (2020) Prevalence of Diabetes Recorded in Mainland China Using 2018 Diagnostic Criteria from the American Diabetes Association: National Cross Sectional Study. BMJ, 369, m997. https://doi.org/10.1136/bmj.m997

[7] Atlas, D. (2017) IDF Diabetes Atlas. 8th Edition, International Diabetes Federation, Brussels.

[8] Ruiz-Ortega, M., et al. (2020) Special Issue "Diabetic Nephropathy: Diagnosis, Prevention and Treatment.” Multidisciplinary Digital Publishing Institute, Basel. 
https://doi.org/10.3390/jcm9030813

[9] Galešić, K., et al. (2009) Dijabetička nefropatija i primarne bolesti glomerula. Liječnički Vjesnik, 131, 141-145.

[10] Wągrowska-Danilewicz, M. and Danilewicz, M. (2015) Spectrum of Biopsy-Proven Renal Diseases in Patients with Type 2 Diabetes Mellitus. A Single Center Study. Polish Journal of Pathology, 66, 361-367. https://doi.org/10.5114/pjp.2015.57247

[11] Ritz, E., Zeng, X.-X. and Rychlík, I. (2011) Clinical Manifestation and Natural History of Diabetic Nephropathy. Diabetes and the Kidney, 170, 19-27. https://doi.org/10.1159/000324939

[12] Wiggins, R.-C. (2007) The Spectrum of Podocytopathies: A Unifying View of Glomerular Diseases. Kidney International, 71, 1205-1214. https://doi.org/10.1038/sj.ki.5002222

[13] Mak, S., et al. (1997) Clinical Predictors of Non-Diabetic Renal Disease in Patients with Non-Insulin Dependent Diabetes Mellitus. Nephrology, Dialysis, Transplantation: Official Publication of the European Dialysis and Transplant Association-European Renal Association, 12, 2588-2591. https://doi.org/10.1093/ndt/12.12.2588

[14] Kasinath, B.S., et al. (1983) Nondiabetic Renal Disease in Patients with Diabetes Mellitus. The American Journal of Medicine, 75, 613-617. https://doi.org/10.1016/0002-9343(83)90442-4

[15] Zhuo, L., Ren, W.W., Li, W.G., Zou, G.M. and Lu, J.H. (2013) Evaluation of renal Biopsies in Type 2 Diabetic Patients with Kidney Disease: A Clinicopathological Study of 216 Cases. International Urology and Nephrology, 45, 173-179. https://doi.org/10.1007/s11255-012-0164-6

[16] Chong, Y.-B., et al. (2012) Clinical Predictors of Non-Diabetic Renal Disease and Role of Renal Biopsy in Diabetic Patients with Renal Involvement: A Single Centre Review. Renal Failure, 34, 323-328. https://doi.org/10.3109/0886022X.2011.647302

[17] Chang, T.I., et al. (2011) Renal Outcomes in Patients with Type 2 Diabetes with or without Coexisting Non-Diabetic Renal Disease. Diabetes Research and Clinical Practice, 92, 198-204. https://doi.org/10.1016/j.diabres.2011.01.017

[18] Soni, S.S., et al. (2006) Non Diabetic Renal Disease in Type 2 Diabetes Mellitus. Nephrology, 11, 533-537. https://doi.org/10.1111/j.1440-1797.2006.00681.x

[19] Pham, T.T., et al. (2007) Prevalence of Nondiabetic Renal Disease in Diabetic Patients. American Journal of Nephrology, 27, 322-328. https://doi.org/10.1159/000102598

[20] Bi, H., Chen, N.L., Ling, G.H., Yuan, S.G., Huang, G.X. and Liu, R.H. (2011) Nondiabetic Renal Disease in Type 2 Diabetic Patients: A Review of Our Experience in 220 Cases. Renal Failure, 33, 26-30. https://doi.org/10.3109/0886022X.2010.536292

[21] Tone, A., et al. (2005) Clinical Features of Non-Diabetic Renal Diseases in Patients with Type 2 Diabetes. Diabetes Research and Clinical Practice, 69, 237-242. https://doi.org/10.1016/j.diabres.2005.02.009

[22] Horvatic, I., et al. (2014) Non-Diabetic Renal Disease in Croatian Patients with Type 2 Diabetes Mellitus. Diabetes Research and Clinical Practice, 104, 443-450.

https://doi.org/10.1016/j.diabres.2014.03.016

[23] Wilfred, D.C., et al. (2013) Nondiabetic Renal Disease in Type 2 Diabetes Mellitus Patients: A Clinicopathological Study. Journal of Laboratory Physicians, 5, 94. https://doi.org/10.4103/0974-2727.119850

[24] Yenigun, E., et al. (2015) Non-Diabetic Renal Disease in Diabetes Mellitus: Clinical Features and Renal Biopsy Findings. Hippokratia, 19, 148. 
[25] Mondal, M.C., et al. (2019) Non Diabetic Renal Disease in Patients with Type 2 Diabetes Mellitus with Proteinuria. Journal of Biosciences and Medicines, 7, 68. https://doi.org/10.4236/jbm.2019.712007

[26] Bermejo, S., et al. (2020) Risk Factors for Non-Diabetic Renal Disease in Diabetic Patients. Clinical Kidney Journal, 13, 380-388. https://doi.org/10.1093/ckj/sfz177

[27] Fan, J.Z. and Wang, R. (2018) Non-Diabetic Renal Disease in Patients with Type 2 Diabetes: A Single Centre Study. Internal Medicine Journal, 48, 451-456. https://doi.org/10.1111/imj.13708

[28] Liang, S., et al. (2014) Clinical Factors Associated with the Diagnosis and Progression of Diabetic Nephropathy. Cell Biochemistry and Biophysics, 70, 9-15. https://doi.org/10.1007/s12013-014-9892-9

[29] Inzucchi, S.E. (2012) Diagnosis of Diabetes. New England Journal of Medicine, 367, 542-550. https://doi.org/10.1056/NEJMcp1103643

[30] Association, A.D. (2014) Diagnosis and Classification of Diabetes Mellitus. Diabetes Care, 37, S81-S90. https://doi.org/10.2337/dc14-S081

[31] Levey, A.S., et al. (2009) A New Equation to Estimate Glomerular Filtration Rate. Annals of Internal Medicine, 150, 604-612. https://doi.org/10.7326/0003-4819-150-9-200905050-00006

[32] Tervaert, T.W.C., et al. (2010) Pathologic Classification of Diabetic Nephropathy. Journal of the American Society of Nephrology, 21, 556-563. https://doi.org/10.1681/ASN.2010010010

[33] Colvin, R.B., Kambham, N., et al. (2011) Glomerular Diseases In: Diagnostic Pathology Kidney Diseases, Amirsys Publishing, Inc., Salt Lake City, 2.2-358.

[34] Prakash, J., et al. (2015) Non-Diabetic Renal Disease in Type 2 Diabetes Mellitus: Study of Renal-Retinal Relationship. Indian Journal of Nephrology, 25, 222. https://doi.org/10.4103/0971-4065.144420

[35] Soleymanian, T., et al. (2015) Non-Diabetic Renal Disease with or without Diabetic Nephropathy in Type 2 Diabetes: Clinical Predictors and Outcome. Renal Failure, 37, 572-575. https://doi.org/10.3109/0886022X.2015.1007804

[36] Mou, S., et al. (2010) Prevalence of Non-Diabetic Renal Disease in Patients with Type 2 Diabetes. Diabetes Research and Clinical Practice, 87, 354-359. https://doi.org/10.1016/j.diabres.2009.11.012

[37] Zwi, L.J., et al. (2014) Non-Diabetic Renal Diseases in a Multi-Ethnic New Zealand Cohort with Type 2 Diabetes Mellitus: Clinical and Histopathological Features. Pathology, 46, 424-432. https://doi.org/10.1097/PAT.0000000000000135

[38] Sharma, S.G., et al. (2013) The Modern Spectrum of Renal Biopsy Findings in Patients with Diabetes. Clinical Journal of the American Society of Nephrology, 8, 17181724. https://doi.org/10.2215/CJN.02510213

[39] Wong, T.Y.H., et al. (2002) Renal Outcome in Type 2 Diabetic Patients with or without Coexisting Nondiabetic Nephropathies. Diabetes Care, 25, 900-905. https://doi.org/10.2337/diacare.25.5.900

[40] Bermejo, S., et al. (2016) Factores predictivos de nefropatía no diabética en pacientes diabéticos. Utilidad de la biopsia renal. Nefrologia, 36, 535-544. https://doi.org/10.1016/j.nefro.2016.06.006

[41] Liu, M.y., et al. (2014) Validation of a Differential Diagnostic Model of Diabetic Nephropathy and Non-Diabetic Renal Diseases and the Establishment of a New Diagnostic Model. Journal of Diabetes, 6, 519-526. https://doi.org/10.1111/1753-0407.12150 
[42] Thomas, M.C., et al. (2006) The Epidemiology of Hemoglobin Levels in Patients with Type 2 Diabetes. American Journal of Kidney Diseases, 48, 537-545.

https://doi.org/10.1053/j.ajkd.2006.06.011

[43] Cotroneo, P., et al. (2000) Blunted Erythropoietin Response to Anemia in Patients with Type 1 Diabetes. Diabetes/Metabolism Research and Reviews, 16, 172-176. https://doi.org/10.1002/1520-7560(200005/06)16:3<172::AID-DMRR117>3.0.CO;2$\underline{D}$

[44] Mehdi, U. and Toto, R.D. (2009) Anemia, Diabetes, and Chronic Kidney Disease. Diabetes Care, 32, 1320-1326. https://doi.org/10.2337/dc08-0779

[45] Tuttle, K.R., et al. (2014) Diabetic Kidney Disease: A Report from an ADA Consensus Conference. American Journal of Kidney Diseases, 64, 510-533.

https://doi.org/10.1053/j.ajkd.2014.08.001 